\title{
Building a bioartificial heart: Obstacles and opportunities
}

\author{
Martha S. Lundberg, PhD, J. Timothy Baldwin, PhD, and Denis B. Buxton, $\mathrm{PhD}$
}

\footnotetext{
From the Division of Cardiovascular Sciences, National Heart, Lung, and Blood Institute, Bethesda, Md.

The authors are employees of the National Heart, Lung, and Blood Institute of the National Institutes of Health. The comments expressed here are those of the authors, and do not reflect official positions of the National Heart, Lung, and Blood Institute or National Institutes of Health.

Received for publication Aug 30, 2016; revisions received Oct 5, 2016; accepted for publication Oct 8, 2016; available ahead of print Jan 27, 2016.

Address for reprints: Denis B. Buxton, PhD, Division of Cardiovascular Sciences, National Heart, Lung, and Blood Institute, 6701 Rockledge Dr, Bethesda, MD 20817 (E-mail: db225a@nih.gov).

J Thorac Cardiovasc Surg 2017;153:748-50

$0022-5223 / \$ 0.00$

Published by Elsevier Inc. on behalf of The American Association for Thoracic Surgery http://dx.doi.org/10.1016/j.jtcvs.2016.10.103
}

The growing numbers of patients with heart failure, both in the United States and around the globe, has led to a pressing need for new therapies. Heart transplantation is a very restricted option due to the limited number of donor hearts available-currently approximately 2800 per year in the United States. Transplant patients also have to take immunosuppressive drugs for the rest of their lives. Ventricular assist devices, used either as bridge to transplant or destination therapy, can prolong patient survival, but present a number of complications, including thrombosis, stroke, and infection. This need could be met by an artificial heart with better biocompatibility than the currently used ventricular assist devices, potentially made immunocompatible by the use of autologous or human leukocyte antigen (HLA) matched allogeneic cells. A number of research laboratories have been working toward this lofty goal.

A recent article describing a light-guided tissue-engineered swimming artificial stingray ${ }^{1}$ (Figure 1) provided impressive proof of concept for the use of cardiomyocytes in a contractile biohybrid machine. The ray was fabricated by seeding dissociated rat cardiomyocytes infected with a gene vector to make them respond to light on a silicone body enclosing a microfabricated gold skeleton. Serpentine patterning of the muscle cells leads to sequential muscle activation in response to light stimulation resulting, in turn, in coordinated undulatory swimming. The gold skeleton obviates the need for a second set of antagonistic muscle cells, instead rebounding once the muscle cells stop contracting.

The robot stingray has attracted a lot of interest in the scientific community as a potential early step toward a bioartificial heart ${ }^{2}$ because some of the design concepts used in the ray could be relevant to more complex structures. For example, genetically encoded bioactuators could be used to control activation of contraction. The use of serpentine muscle patterning to promote sequential muscle activation could be adapted to provide the organized contraction needed to pump blood in a bioartificial heart. The use of a compressible skeleton that rebounds to diastole after systolic contraction may also be a theme to be explored.

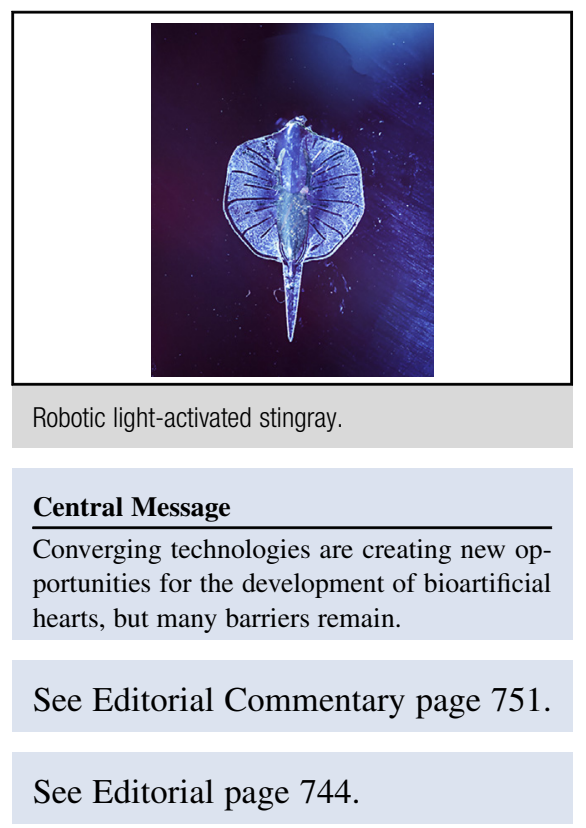

It is instructive to place this research in the context of the current status of work toward development of a bioartificial heart, including the limitations of current technologies, future directions that the ray could facilitate, and the opportunities and obstacles that exist.

\section{CURRENT STATUS OF THE BIOARTIFICIAL HEART}

Although there has been widespread interest in the use of regenerative medicine to improve cardiac function both in acute myocardial infarction and in chronic heart failure, the clinical benefit has been modest and variable between clinical trials, with some showing no benefit. The failure to achieve clinical success following positive results obtained in preclinical models is likely a reflection of patient comorbidities such as diabetes, metabolic syndrome, and hypertension that may influence the efficacy of cell therapy. This has stimulated interest in tissue engineering approaches ranging from coinjection of artificial matrices to the development of tissue patches and ultimately to bioartificial hearts.

Much of the current work on bioartificial heart development has focused on the use of decellularized hearts in which detergent is used to strip cells from a heart followed by recellularization. The scaffolds retain the macro- and microarchitecture, vasculature, and biochemical cues for cellular adherence, proliferation, and differentiation. ${ }^{3}$ Experiments using rat hearts have shown that hearts can be 
successfully recellularized and will contract to develop pressure, although at much lower levels than needed physiologically. ${ }^{3}$ Decellularization has also been carried out successfully on porcine and human hearts. One potential limitation of the technology is that the number of socalled normal scaffolds available is limited. As mentioned previously, healthy donor hearts are used for transplantation and scaffolds derived from diseased or damaged hearts are compromised by scarring and remodeling. The use of xenogeneic scaffolds such as porcine-derived scaffolds could alleviate the shortfall. ${ }^{3}$

\section{FUTURE DIRECTIONS}

The development of a bioartificial heart from scratch using a synthetic scaffold and cardiac cells is very challenging due to the complexity of the myocardial structure, which uses ventricular torsion to eject $60 \%$ of blood within the left ventricular cavity while the cardiomyocytes driving the pumping shorten by only $15 \%$. The increasing availability and sophistication of three-dimensional (3D) printing offers a tantalizing vision that complex structures such as a functional bioartificial heart may be on the horizon. 3D printing is already being used to make models of hearts based on cardiac imaging that aid surgeons in surgical planning. Printing a cardiac scaffold that mimics decellularized hearts using cell-instructive materials is likely to be a reachable goal in the relatively near future as the technology continues to evolve. Recent advances include bioprinting of human cardiomyocyte progenitor cells into alginate scaffolds to form cardiac constructs, ${ }^{4}$ and fabricating cardiac patches containing human vascular endothelial cells and human mesenchymal stromal cells. ${ }^{5}$ Bioprinting with incorporation of cells into the scaffold during the printing process is thus a possibility in the longer term. The work from Park and colleagues ${ }^{1}$ suggests another possibility: bioprinting a hybrid scaffold, perhaps containing a compressible scaffold such as that seen in the ray, and incorporating muscle patterning to promote spatial organization of contraction for pumping. Whether this type of hybrid design can improve on nature by providing a simplified design while retaining the efficient pumping of the native heart remains to be seen.

\section{OPPORTUNITIES AND OBSTACLES}

New technologies such as induced pluripotent stem cells (iPSCs), 3D printing, and advances in in silico modeling are providing new directions toward a bioartificial heart. However, a number of considerable technological barriers remain before a bioartificial heart can move toward preclinical testing and eventually to clinical application.

\section{iPSCs}

The development of iPSCs, which can be differentiated to cardiomyocytes and other cardiac cell types, opens up new

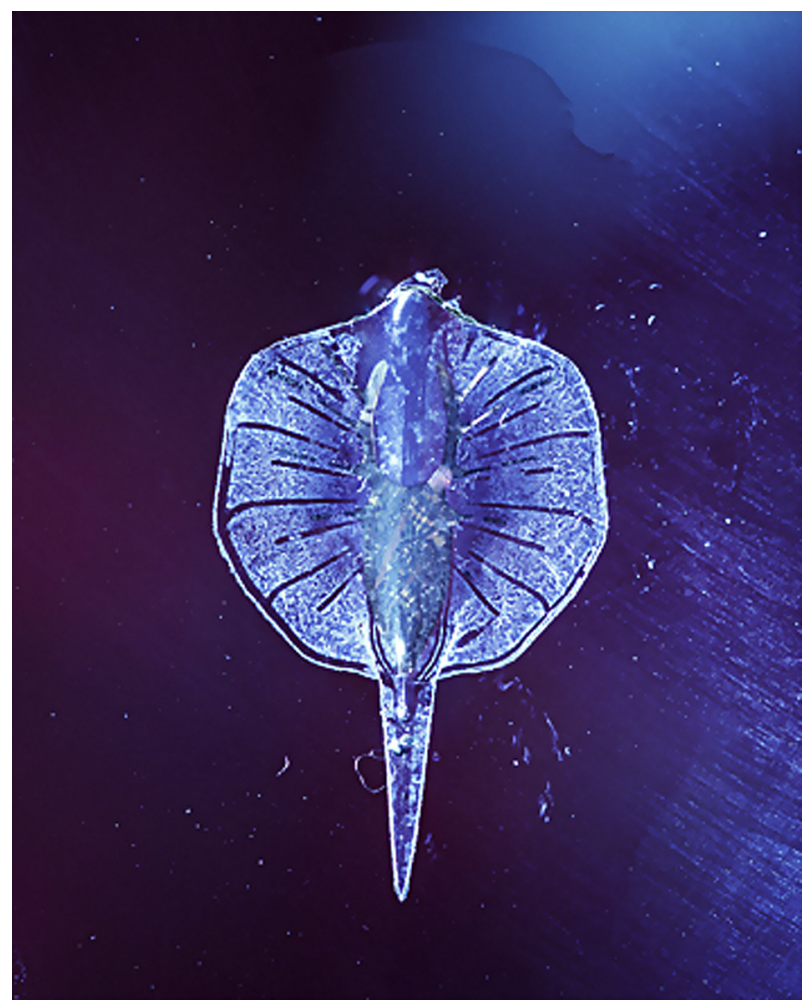

FIGURE 1. Robotic light-activated stingray manufactured from cardiomyocytes, and gold scaffold encapsulated in a silicone hydrogel body. Reprinted from Park and colleagues ${ }^{1}$ with permission from the American Association for the Advancement of Science.

avenues for the development of bioartificial hearts. The potential for using autologous iPSCs derived from a patient's own blood or skin offers the attractive possibility of building an immune-compatible heart for each patient. This could result in a patient not needing immunosuppression, although questions remain about whether autologous iPSCs and their derived products are completely immunocompatible. Alternatively, large cell banks of iPSCs from multiple donors could provide allogeneic cells that could be HLAmatched to most individuals, creating economy of scale by generating enough cells for multiple hearts from a single donor culture. The availability of HLA-matched allogeneic cells would also obviate the need for extended expansion of autologous cells to obtain the large number of cells needed, and would be more feasible from a manufacturing and economic standpoint. However, a significant scale-up from current methods will be required for a heart for human applications. The robotic stingray was built using approximately 200,000 rat cardiomyocytes derived from dissociated neonatal hearts. Decellularized rat hearts were recellularized using approximately $10^{8}$ neonatal rat cardiomyocytes. Scaling up from rat to a human heart weighing approximately $300 \mathrm{~g}$ would obviously require many more cells: approximately $5 \times 10^{10}$. The need to develop scalable technologies for generating large numbers of functional 
cells for the use in a bioartificial heart is a significant challenge. In addition, the generation of additional cell types, including fibroblasts and vascular cells, is likely to be needed to generate a bioartificial heart.

\section{D Printing}

Printers have been increasingly used for biomedical applications during the past decade, and especially for the field of tissue engineering. 3D printing is a promising technique for the development of cardiac patches, valves, and blood vessels, but is still in its infancy. Using imaging data from whole hearts, Hinton and colleagues ${ }^{6}$ used a thermoreversible support bath to bioprint an embryonic heart with complex internal and external anatomical structures, but appropriate vascularization of the heart was absent. Factors that need to be optimized include mechanical strength and integrity and the balance between the mechanical properties of bioprinted hydrogels and the effect on cellular function. The microenvironment, matrix components, stiffness, and incorporation of biomolecules that control differentiation of cells also need to be controlled. Improvement of hydrogel crosslinking in situ is a major challenge, and a more realistic near-term goal in organ printing is to develop miniorgans or constructs with the minimal functional requirements to assist a failing heart. Proof-of-concept studies have demonstrated the feasibility of bioprinting live cells and maintaining their viability in small organoids, but scaling up to an organ the size of a human heart will be extremely challenging. The complexity of the heart, which would require endothelial cells and smooth muscle cells to supply vasculature and fibroblasts to maintain extracellular matrix, provides further challenges in optimizing cell patterning to generate a functional construct. Finally, for any successful clinical application of a bioartificial heart, the blood-contacting biomaterials used in its construction must be designed and developed to be thrombo- and calcification-resistant after implantation.

\section{In Silico Modeling}

As finite element modeling, computational fluid dynamics, and fluid-structure interaction software capabilities and techniques continue to improve and become more sophisticated, such tools could be customized and applied to facilitate the development of a bioartificial heart. Finite element modeling could be used to evaluate various structural components, such as the design of synthetic scaffolds. Electromechanical models could be used to simulate contraction of the cardiomyocytes used in the bioartificial hearts, and sophisticated fluid-structure interaction software can potentially model the contraction and relaxation of the cardiac tissue of a bioartificial heart and the resulting blood flow produced. Such analyses could lead to novel designs of artificial structures for a bioartificial heart.

\section{Maturation of Constructs}

Cardiomyocytes differentiated from iPSCs are immature-resembling fetal or neonate cardiomyocytes-and this contributes to the low mechanical efficiency of recellularized hearts. For recellularized hearts to become more efficient, maturation of the constructs in a bioreactor with mechanical and electrical stimulation of the construct is needed. Maintaining sterility and appropriate conditions for survival and maturation of constructs for several months for a construct the size of a human heart will require significant advances in bioreactor capabilities. An alternative approach that could shorten the in vitro maturation period would be to implant a partially matured construct and combine mechanical support with in vivo maturation, allowing the implanted organ to take over eventually. ${ }^{3}$ Within this context, the Department of Defense has released a new funding opportunity announcement for the purpose of establishing a state-of-the-art, end-to-end, sustainable Manufacturing Innovation Institute in support of advanced tissue biofabrication manufacturing.

\section{CONCLUSIONS}

The development of a robotic stingray represents a technical tour de force and proof of principle for the use of cardiomyocytes to form contractile structures. Moving from this small and relatively simple structure to the complexities and scale of a human heart will require that a number of technical challenges be overcome.

\section{Conflicts of Interest Statement}

Authors have nothing to disclose with regard to commercial support.

\section{References}

1. Park SJ, Gazzola M, Park KS, Park S, Di Santo V, Blevins EL, et al. Phototactic guidance of a tissue-engineered soft-robotic ray. Science. 2016;353:158-62.

2. Pennisi E. ROBOTICS. Heartmaker's next step: a ray 'biohybrid'. Science. 2016; 353:110-1.

3. Moser PT, Ott HC. Recellularization of organs: what is the future for solid organ transplantation? Curr Opin Organ Transplant. 2014;19:603-9.

4. Zhang YS, Yue K, Aleman J, Mollazadeh-Moghaddam K, Bakht SM, Yang J, et al. 3D bioprinting for tissue and organ fabrication. Ann Biomed Eng. 2017;45:148-63.

5. Ye L, Basu J, Zhang J. Fabrication of a myocardial patch with cells differentiated from human-induced pluripotent stem cells. Methods Mol Biol. 2015;1299: 103-14.

6. Hinton TJ, Jallerat Q, Palchesko RN, Park JH, Grodzicki MS, Shue HJ, et al. Three-dimensional printing of complex biological structures by freeform reversible embedding of suspended hydrogels. Sci Adv. 2015;1:e1500758. 